\title{
Versatile simplified physical model for parallel aligned liquid crystal devices
}

\section{Andrés Márquez, Francisco J. Martínez-Guardiola, Jorge Francés, Sergio Bleda, Mariela L. Alvarez, et al.}

Andrés Márquez, Francisco J. Martínez-Guardiola, Jorge Francés, Sergio Bleda, Mariela L. Alvarez, Inmaculada Pascual, Sergi Gallego, Augusto Beléndez, "Versatile simplified physical model for parallel aligned liquid crystal devices," Proc. SPIE 10751, Optics and Photonics for Information Processing XII, 1075106 (7 September 2018); doi: 10.1117/12.2321054

SPIE Event: SPIE Optical Engineering + Applications, 2018, San Diego, California, United States 


\title{
Versatile simplified physical model for parallel aligned liquid crystal devices
}

\author{
Andrés Márquez ${ }^{1,2, *}$, Francisco J. Martínez-Guardiola ${ }^{1,2}$, Jorge Francés ${ }^{1,2}$, Sergio Bleda ${ }^{1,2}$, Mariela L. \\ Alvarez $^{\mathbf{1 , 2}}$, Inmaculada Pascual ${ }^{2,3}$, Sergi Gallego ${ }^{1,2}$, Augusto Beléndez ${ }^{1,2}$ \\ ${ }^{1}$ Dept. de Física, Ing. de Sistemas y T. Señal, Univ. de Alicante, Ap. 99, E-03080, Alicante, Spain \\ ${ }^{2}$ I.U. Física Aplicada a las Ciencias y las Tecnologías U. de Alicante, Ap. 99, E-03080, Alicante, Spain \\ ${ }^{3}$ Dept. de Óptica, Farmacología y Anatomía, Univ. de Alicante, Ap. 99, E-03080, Alicante, Spain \\ corresponding autor: andres.marquez@ua.es
}

\begin{abstract}
Liquid crystal on silicon (LCoS) displays have become the most attractive microdisplays for all sort of spatial light modulation applications. Among the different LCoS technologies, parallel aligned LCoS (PA-LCoS) are especially interesting. They offer unique capabilities as spatial light modulators (SLM), since they enable phase-only modulation without coupled amplitude modulation, and with millions of addressable pixels. In this work we evaluate and demonstrate the various capabilities offered by a novel and simplified physical model for parallel aligned liquid crystal devices (PA-LC), thus also applicable to PA-LCoS microdisplays. The model provides the voltage dependent retardance values, necessary for spatial light modulation applications, for a very wide range of incidence angles and any wavelength in the visible. First, it needs to be calibrated through a reverse-engineering approach. In this calibration, the values obtained for two of the three parameters provide the correct values for important internal properties of these devices related with the birefringence, cell gap and director profile. Therefore, the proposed model can be used as a means to inspect internal physical properties of the cell. Last but not least the model is useful to simulate the retardance for novel PA-LC devices as a function of the LC compound and cell gap. Therefore, it is not only a reverse-engineering model but it also constitutes an analytical alternative to the usual numerical approaches for PA-LC devices design and construction.
\end{abstract}

Keywords: Liquid-crystal devices, Parallel-aligned, Birefringence, Spatial light modulators, Displays.

\section{INTRODUCTION}

There is an interest in proposing simplified analytical models for liquid crystal (LC) devices ${ }^{[1][2]}$. They enable a more direct access to the analysis of the capabilities offered by these technologies in a wide variety of applications. In spatial light modulation applications, one of the most widespread technologies has become the parallel-aligned liquid crystal on silicon microdisplays (PA-LCoS $)^{[3][4][5][6]}$. They enable phase-only modulation of light wavefronts without coupled amplitude modulation, with millions of addressable pixels, and with very small pixel sizes, smaller than $10 \mu \mathrm{m}$ in many cases. They are also easy devices to model, at least in principle, since they are equivalent to variable linear retarders whose linear retardance is tuned by the applied voltage. Proper characterization of their linear retardance ensures optimal performance in applications. In previous works, we proposed time-averaged Stokes polarimetry ${ }^{[7]}$ as an advanced technique which enables robust linear retardance characterization in the presence of flicker, which is a typical degradation phenomenon found in most of LCoS devices ${ }^{[8][9][10]}$.

In a more recent paper ${ }^{[11]}$ we proposed a semiphysical model able to describe the variation of the tilt angle of the molecules as a function of the voltage. The model is highly simplified, however we demonstrated ${ }^{[12][13]}$ that it retained the adequate level of the physics: when fitting the model, for two of its three parameters the values obtained are equal to the physical values of corresponding internal properties of the device and the LC. Two parameters are a combination of the LC indexes ordinary and extraordinary, $n_{o}$ and $n_{e}$, together with the cell gap $d$. These parameters are $O P L=d n_{o}$ and $O P D=d \Delta n$, which do not depend on the applied voltage and we call off-state parameters. The other parameter is the equivalent tilt angle $\alpha(V)$ of the molecules, which depends on the applied voltage. The values obtained in the fitting

Optics and Photonics for Information Processing XII, edited by Abdul A. S. Awwal, Khan M. Iftekharuddin, Mireya García Vázquez, Andrés Marquez, Víctor H. Diaz-Ramirez Proc. of SPIE Vol. 10751

$1075106 \cdot$ C 2018 SPIE · CCC code: 0277-786X/18/\$18 · doi: 10.1117/12.2321054 
procedure for $O P D$ and $\alpha(V)$ correspond to their true values ${ }^{[12]}$. This is not the case for $O P L$, however we showed ${ }^{[13]}$ that large variations in this parameter do not have much effect on the retardance value calculated. This enables to use the model to simulate the retardance for novel PA-LC devices as a function of the LC compound and cell gap.

Now, in this paper, we will show some of the possibilities offered by this semiphysical model. We will show that it enables to calculate the working configurations (incidence angle, applied voltage, illumination wavelength) where the wavelength or incidence angle can be tuned accordingly to produce the needed absolute retardance value or to generate the retardance dynamic range needed by the application in mind.

\section{SIMPLIFIED SEMIPHYSICAL MODEL}

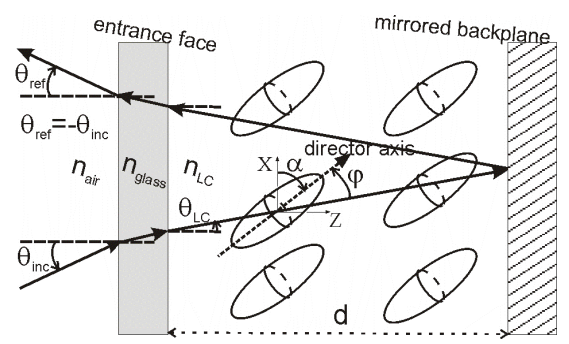

Figure 1. Diagram for the PA-LC cell considered in the model proposed.

For the sake of completeness of this work, we present the simplified semiphysical model, which is further detailed in the paper where it was initially proposed ${ }^{[11]}$. We present in Fig. 1 its general diagram for a reflective cell with a cell gap $d$. Incidence plane and $\mathrm{LC}$ director are along the $\mathrm{XZ}$ plane. $\mathrm{LC}$ molecules have their director axis (optical axis) aligned at an angle $\varphi$ with respect to the traversing light beam direction. $\theta_{L C}$ is the refraction angle in the LC medium. The director axis tilts an angle $\alpha$ with respect to the entrance face as a function of the applied voltage $\mathrm{V}$. This is the only voltage dependent magnitude, i.e. $\alpha(V)$. At the backplane the light beam is reflected and a second passage is produced across the LC layer whose effect is equivalent to a forward propagation at an angle $-\theta_{i n c}$. Apart from the on-state parameter $\alpha(V)$, the model has two voltage independent parameters, which we call off-state parameters. They are the optical path length for the ordinary component, $O P L=d n_{o}$, and the optical path difference between extraordinary and ordinary components, $O P D=d \Delta n$, in which $n_{o}$ and $n_{e}$ are respectively the LC indexes ordinary and extraordinary. The main expression produced by the $\operatorname{model}^{[11]}$ is,

$$
\Gamma=\frac{2 \pi}{\lambda} \frac{O P L}{\cos \theta_{L C}}\left[\frac{1+(O P D / O P L)}{1+(O P D / O P L) \cos ^{2} \varphi}-1\right]
$$

According to Fig. 1, angle $\varphi$ is given by,

$$
\varphi\left(\theta_{i n c}, V\right)=\frac{\pi}{2}+\alpha(V) \mp \theta_{L C}\left(\theta_{i n c}\right)
$$

, where the minus (plus) sign applies for the forward (backward) passage. The total retardance in the PA-LCoS is given by the addition of the forward and backward retardances. In the case of normal incidence and LC director axis parallel to the entrance face, then Eq. (1) simplifies into the well-known expression $\Gamma=2 \pi d \Delta n / \lambda$.

We note that in our previous works ${ }^{[11][12]}$, we also evaluated the exact expressions for a homogeneous uniaxial anisotropic plate ${ }^{[14][15]}$. A first approximation shared by both models is that they do not consider that the tilt angle of the LC director changes across the cell. Our model further disregard the existence of double refraction. We obtained that both simplified models provide the same level of predictive capability, with the benefit that our model produces a much simpler expression and reduces the number of parameters when compared with these exact expressions. Additionally, 
when fitting the parameters, our model converges to a unique solution, whose values are equal to the real physical values in two of its three parameters ${ }^{[12]}$ : OPD and tilt angle $\alpha(V)$. This was not the case with the exact expressions

\section{EXPERIMENT AND CALIBRATION}

As PA-LC device of study, we consider a commercially available PA-LCoS microdisplay, model PLUTO distributed by the company HOLOEYE. It is a nematic liquid crystal filled, with 1920x1080 pixels and 0.7" diagonal, whose backplane is digitally addressed ${ }^{[16]}$. By means of a RS-232 interface and its corresponding provided software, we can configure the modulator for different applications and wavelengths. Besides, different pulse width modulation (PWM) addressing schemes (digital addressing sequences) can be generated by the driver electronics ${ }^{[8][16]}$. We consider the configuration file "5-5 633 2pi linear", provided by the vendor. As verified in previous papers ${ }^{[16]}$, it shows a smaller magnitude of flicker than other electrical sequences configuration files, and it is adapted to produce a $2 \pi$ radians linear phase dynamic range at $633 \mathrm{~nm}$ and for perpendicular incidence.

The time-averaged Stokes polarimetric measurements are obtained with a Stokes polarimeter, model PAX5710VIS-T distributed by the company THORLABS ${ }^{[7]}$. The polarimeter averaging time considered, $600 \mathrm{~ms}$, is much larger than actually needed to obtain fully stable and repeatable SOP measurements. Note that the time period (frequency) for the fluctuations in our PA-LCoS device is $8.66 \mathrm{~ms}(120 \mathrm{~Hz})^{[7]}$. Measurements are taken at various angles of incidence $\left(3^{\circ}\right.$, $23^{\circ}, 35^{\circ}$ and $\left.45^{\circ}\right)$ and for three wavelengths $(473,532$ and $633 \mathrm{~nm})$ sampling the visible spectrum. We use the measurements taken at $3^{\circ}$ and $35^{\circ}$ for calibration, and then we use the measurements at $23^{\circ}$ and $45^{\circ}$ to analyse the predictive capability of the semiphysical model.

In Table 1 we show the off-state values measured for the retardance, and in Table 2 we show the OPD and OPL values obtained in the calibration. The figure of merit $\chi^{2}$ to be minimized combines two squared differences: on one hand between theoretical and experimental retardance values normalized by the experimental value, and on the other hand between the theoretical and experimental ratios of the retardance values for the pair of incidence angles considered $\left(3^{\circ}\right.$ and $35^{\circ}$ ) normalized by the experimental ratio. These two normalized squared differences are added up for the three wavelengths and the resultant minimized value for each solution set is shown in row $\chi^{2}$ (second row). We note that we consider $n_{L C}=1.50$ in the fitting procedure ${ }^{[12]}$, which minimized the value of $\chi^{2}$ and which is within the range of physically meaningful values for the average refractive index of the LC layer.

Table 1. Values for the retardance in the off-state.

\begin{tabular}{ccccc}
\hline$\lambda$ & \multicolumn{4}{c}{ Incidence angle } \\
& $3^{\mathrm{o}}$ & $23^{\circ}$ & $35^{\circ}$ & $45^{\circ}$ \\
\hline$(\mathrm{nm})$ & $(\mathrm{deg})$ & $(\mathrm{deg})$ & $(\mathrm{deg})$ & $(\mathrm{deg})$ \\
\hline 633 & 599.5 & 562.4 & 538.3 & 506.5 \\
532 & 802.8 & 771.3 & 718.5 & 703.2 \\
473 & 987.0 & 945.6 & 900.3 & 867.1 \\
\hline
\end{tabular}

Table 2. Fitted OPD and OPL using the off-state retardance measurements for $\theta_{i n c}=3^{\circ}$ and $35^{\circ}$.

\begin{tabular}{cccc}
\hline$\lambda(\mathrm{nm})$ & 633 & 532 & 473 \\
\hline $\operatorname{OPD}(\mu \mathrm{m})$ & 0.5275 & 0.5937 & 0.6489 \\
$\operatorname{OPL}(\mu \mathrm{m})$ & 2.5761 & 2.6064 & 6.8925 \\
\hline
\end{tabular}

Now we fix the OPD and OPL values from Table 2 in the theoretical expressions for the semiphysical model. And we use the on-state retardance experimental measurements at incidences $3^{\circ}$ and $35^{\circ}$ in the fitting procedure, to produce the values for the tilt angle as a function of voltage $\alpha(V)$. The same figure of merit function $\chi^{2}$, previously explained in the off-state calibration, is also applied for the on-state fitting procedure, and the optimization is run independently for each of the gray level (equivalent to applied voltage) values.

In Fig. 2 we show the tilt angle $\alpha(V)$ as a function of the gray level. The tilt angle increases monotonically with the gray level, which reflects that the applied voltage is increasing. We note that the curve is highly nonlinear at lower gray levels 
and reaches maximum values about $50^{\circ}$. According to previous studies ${ }^{[13]}$, where we considered the non-linear tilt angle profile across the cell thickness, we are still within the range where tilt angle profiles across the cell thickness do not saturate in the cell bulk. This is actually the situation when our model shows the best agreement with experiment ${ }^{[12][13]}$.

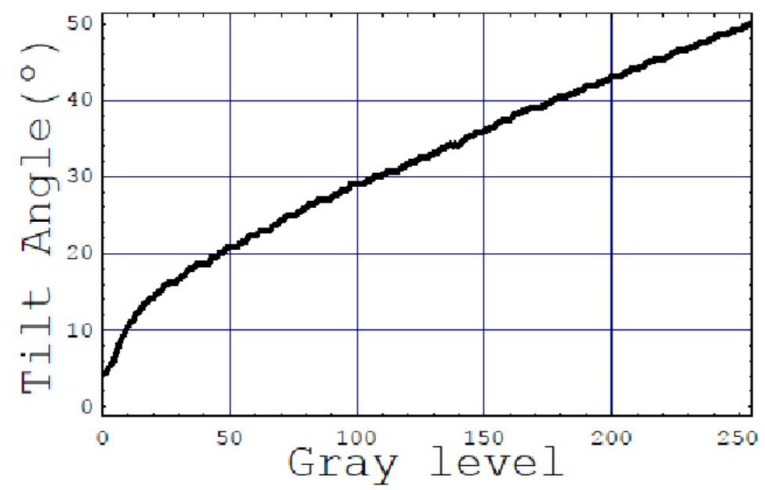

Figure 2. Tilt angle as a function of gray level obtained with the proposed model.

We want to remark that in previous works we have shown that the values obtained for OPD and for $\alpha(V)$ are in very good agreement with the true values ${ }^{[12][13]}$. This means that the model is not a "black-box" but that it is useful to probe into internal properties of the PA-LC devices.

\section{SPECTRAL AND ANGULAR RETARDANCES TO TUNE WORKING CONFIGURATIONS}

We have obtained the values for parameters in the model, thus now the model is ready to provide calculations useful in many applications where PA-LCoS devices are used. In this Section, we apply this versatility of the model to spectral and angular retardance calculations under different working configurations. Since one thing we want to do is show the possibility of spectral calculations, first thing to do is to produce the OPD and OPL dependence with wavelength by applying the sampled values at the three wavelengths considered in the calibration procedure. We fit the extended Cauchy ${ }^{[18]}$ relation $A+B / \lambda^{2}+C / \lambda^{4}$ to the OPD values for the three wavelengths (Table 2). In the case of the OPL, whose values are not related with actual true values, simply a linear interpolation has been applied. In Fig. 3(a) and (b) we show respectively for OPD and OPL the results, where the lines correspond to the interpolation and the points are the three sampling values in Table 2 .
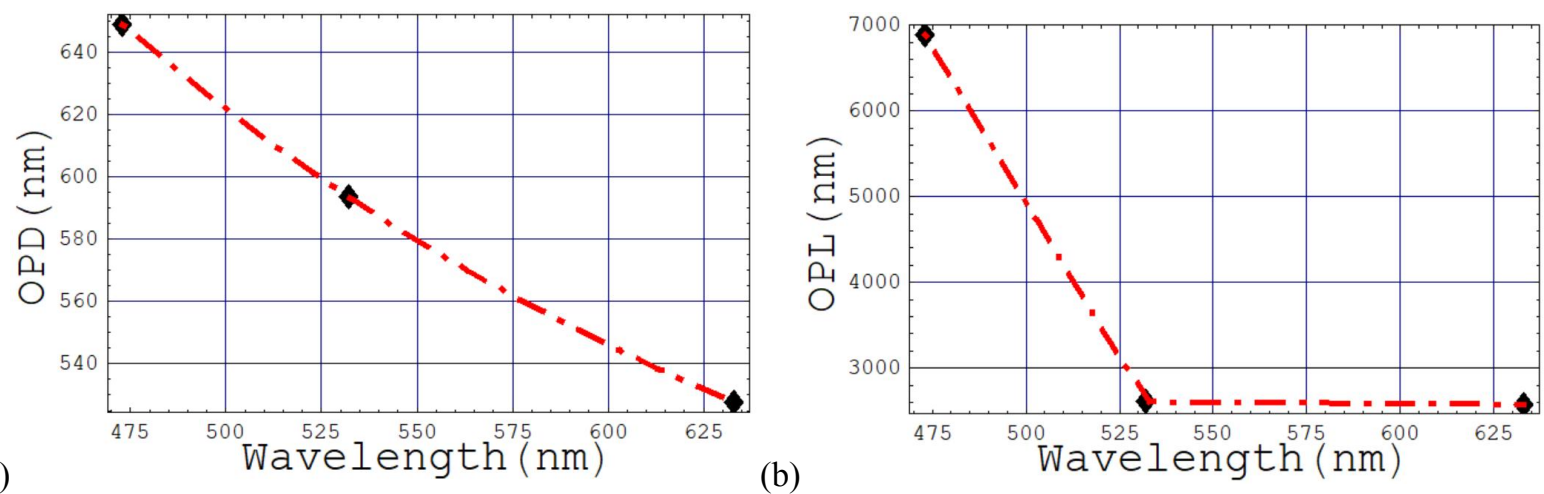

Figure 3. Spectral variation of (a) OPD, and (b) OPL. Calibrated values (diamonds) used to obtain the interpolating functions are shown together with these functions (lines). 
By applying the curves in Fig. 3, the semiphysical model can be used to obtain the retardance across the wavelengths in the visible region of the spectrum and for a wide range of incidences (till $45^{\circ}$ ). The spectral prediction capability of the model opens its applicability, for example, to spectral retardance control in applications such as the one reported by Moreno et al. ${ }^{[19]}$, where they show a system that acts as an optical retarder where the retardance spectral characteristics can be defined at will, and it can be reprogrammed in real time. To this goal, the kind of calculations we show in Fig. 4 are useful to establish the most appropriate working configuration, i.e. angle of incidence and voltage value to be applied. In Fig. 4 we show the retardance dependence with wavelength at three angles of incidence, respectively $0^{\circ}, 25^{\circ}$ and $45^{\circ}$ in (a), (b) and (c). In each plot we show the spectral retardance at various applied gray levels, shown in the legend. The dynamic range at each wavelength, i.e. difference between the maximum and minim retardance value, is shown in Fig. 4(d) for the three incidence angles, shown in the legend. We see that the dynamic range decreases with the angle of incidence. It is larger than $2 \pi$ radians $\left(360^{\circ}\right)$ at a limited range of the spectral range for incidence at $25^{\circ}$ and only at the shortest wavelength at incidence $45^{\circ}$, whereas at normal incidence the whole visible spectrum overcomes this range. In diffractive optics and other applications dynamic ranges larger than $2 \pi$ radians are demanded ${ }^{[4][5][6]}$.
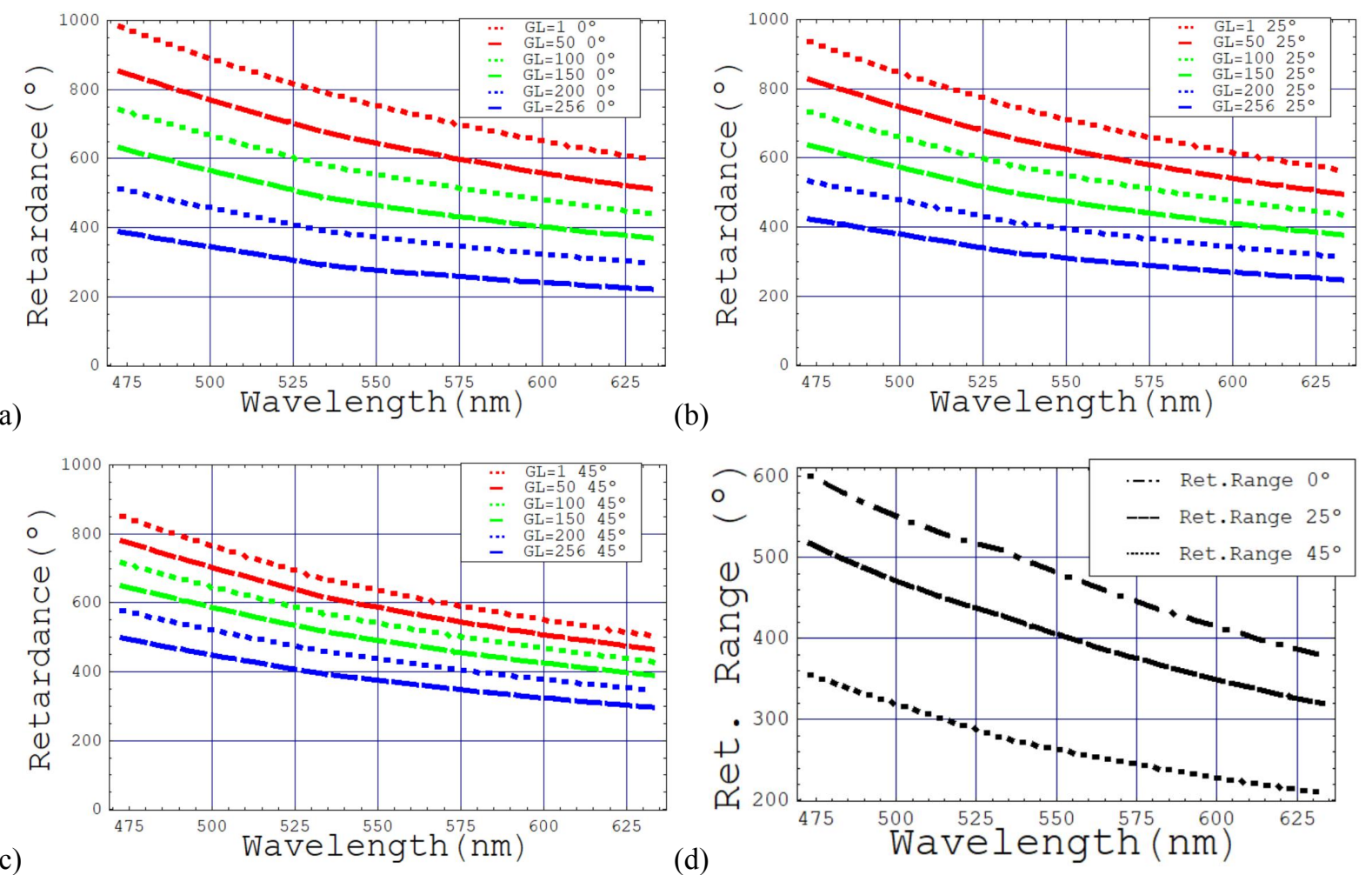

b)

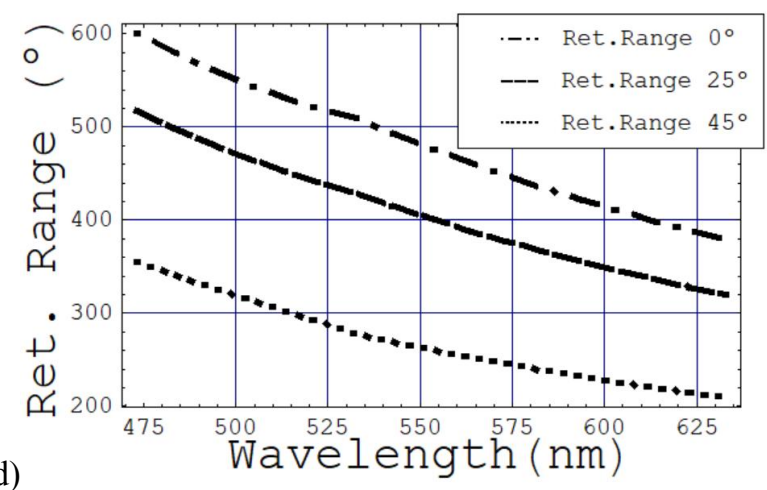

Figure 4. Tilt angle as a function of gray level obtained with the proposed model.

\section{CONCLUSIONS}

We have shown a semiphysical model that eases the applicability of PA-LC devices in spatial light modulation. The model can be used to provide calculations useful in many applications where PA-LCoS devices are used. In particular, we have shown that it enables to calculate the working configurations (incidence angle, applied voltage, illumination wavelength) where the wavelength or incidence angle can be tuned accordingly to produce the needed absolute retardance value or to generate the retardance dynamic range needed by the application in mind. 


\section{ACKNOWLEDGEMENTS}

This work was supported by Ministerio de Economía, Industria y Competitividad (Spain) under projects FIS2017-82919R (MINECO/AEI/FEDER, UE) and FIS2015-66570-P (MINECO/FEDER), by Generalitat Valenciana (Spain) under project PROMETEO II/2015/015 and by Universidad de Alicante under project GRE17-06.

\section{REFERENCES}

[1] K. Lu, B. E. A. Saleh, "Theory and design of the liquid crystal TV as an optical spatial phase modulator," Opt. Eng. 29, 240-246 (1990).

[2] A. Márquez, C. Iemmi, I. Moreno, J. A. Davis, J. Campos, M. J. Yzuel, "Quantitative prediction of the modulation behavior of twisted nematic liquid crystal displays based on a simple physical model," Opt. Eng. 40, 2558-2564 (2001).

[3] S. T. Wu and D. K. Yang, [Reflective Liquid Crystal Displays], John Wiley \& Sons Inc. (2005).

[4] G. Lazarev, A. Hermerschmidt, S. Krüger, and S. Osten, "LCOS Spatial Light Modulators: Trends and Applications," in [Optical Imaging and Metrology: Advanced Technologies], W. Osten and N. Reingand, eds., John Wiley \& Sons (2012).

[5] N. Collings, T. Davey, J. Christmas, D. Chu, and B. Crossland, "The Applications and Technology of PhaseOnly Liquid Crystal on Silicon Devices,” J. Display Technol. 7, 112-119 (2011).

[6] Z. Zhang, Z. You, D. Chu, "Fundamentals of phase-only liquid crystal on silicon (LCOS) devices," Light: Science \& Applications 3, 1-10 (2014).

[7] F.J. Martínez, A. Márquez, S. Gallego, J. Francés, I. Pascual, and A. Beléndez, "Retardance and flicker modeling and characterization of electro-optic linear retarders by averaged Stokes polarimetry," Opt. Lett. 39, 1011-1014 (2014).

[8] A. Hermerschmidt, S. Osten, S. Krüger, and Thomas Blümel, "Wave front generation using a phase-only modulating liquid-crystalbased micro-display with HDTV resolution," Proc. SPIE 6584, 65840E (2007).

[9] A. Lizana, I. Moreno, A. Márquez, E. Also, C. Iemmi, J. Campos, and M.J. Yzuel, "Influence of the temporal fluctuations phenomena on the ECB LCoS performance," Proc. SPIE 7442, 74420G-1 (2009).

[10] J. García-Márquez, V. López, A. González-Vega, E. Noé, "Flicker minimization in an LCoS spatial light Modulator," Opt. Express 20, 8431-8441 (2012).

[11]F. J. Martínez, A. Márquez, S. Gallego, J. Francés, I. Pascual, A. Beléndez, "Effective angular and wavelength modelling of parallel aligned liquid crystal devices," Opt. Lasers Eng. 74, 114-121 (2015).

[12] A. Márquez, J. Francés, F. J. Martínez-Guardiola, S. Gallego, M. L. Álvarez, E. M. Calzado, I. Pascual, A. Beléndez, "Computational SF-FDTD evaluation of simplified tilt-angle models for parallel aligned liquid crystal devices," Opt. Eng. 57, 037110 (2018).

[13] J. Francés, A. Márquez, F. J. Martínez-Guardiola, S. Bleda, S. Gallego, C. Neipp, I. Pascual, A. Beléndez, "Simplified physical modeling of parallel-aligned liquid crystal devices at highly non-linear tilt angle profiles," Opt. Express 26, 12723-12741 (2018).

[14]A. Lien, "Extended Jones matrix representation for the twisted nematic liquid-crystal display at oblique incidence," Appl. Phys. Lett. 57, 2767-2769 (1990).

[15] P. Yeh, C. Gu, [Optics of Liquid Crystal Displays], John Wiley \& Sons Inc. (1999).

[16]F. J. Martínez, A. Márquez, S. Gallego, M. Ortuño, J. Francés, A. Beléndez, and I. Pascual, "Electrical dependencies of optical modulation capabilities in digitally addressed parallel aligned LCoS devices," Opt. Eng. 53, 067104 (2014).

[17]F.J. Martínez, A. Márquez, S. Gallego, M. Ortuño, J. Francés, A. Beléndez, and I. Pascual, "Averaged Stokes polarimetry applied to evaluate retardance and flicker in PA-LCoS devices," Opt. Express 22, 15064-15074 (2014).

[18] J. Li, C-H. Wen, S. Gauza, R. Lu, S-T. Wu, "Refractive Indices of Liquid Crystals for Display Applications," J. Disp. Technol. 1, 51-61 (2005).

[19] I. Moreno, J. V. Carrión, J. L. Martínez, P. García-Martínez, M. M. Sánchez-López, J. Campos, "Optical retarder system with programmable spectral retardance," Opt. Lett. 39, 5483-5486 (2014). 